\title{
Rotation-dependent nonlinear absorption of orbital angular momentum beams in ruby
}

\author{
G. Musarra ${ }^{1}$, K. E. Wilson ${ }^{2}$, D. Faccio ${ }^{1 *}$, AND E. M. Wright 3,2 \\ ${ }^{1}$ School of Physics and Astronomy, University of Glasgow, Glasgow, G12 8QQ, UK. \\ ${ }^{2}$ School of Engineering and Physical Sciences, Heriot-Watt University Edinburgh, EH14 4AS, United Kindom. \\ ${ }^{3}$ College of Optical Sciences, University of Arizona, Tucson, Arizona 85721, USA \\ *Corresponding author: daniele.faccio@glasgow.ac.uk
}

Compiled May 26, 2018

\begin{abstract}
We investigate the effect of a rotating medium on orbital angular momentum (OAM) carrying beams by combining a weak probe beam shifted in frequency relative to a strong pump beam. We show how the rotational Doppler effect modifies the light-matter interaction through the external rotation of the medium. This interaction leads to an absorption that increases with the mechanical rotation velocity of the medium and with a rate that depends on the orbital angular momentum of the light beam. (- 2018 Optical Society of America
\end{abstract}

OCIS codes: (190.5940) Self-action effects; (080.4865) Optical vortices; (260.1180) Crystal optics; (190.4223) Nonlinear wave mixing.

http://dx.doi.org/10.1364/ao.XX.XXXXXX

The rotational Doppler effect is the rotational counterpart of the translational Doppler effect that arises when the source is in relative rotation with respect to the observer. Originally introduced by Garetz et al. [1], this phenomenon is typically observed with angular momentum carrying beams propagating in a rotating medium $[2,3]$ : in this case the rotation induces a frequency shift in the observed beam that is proportional to both the rotational rate and the angular momentum state [4], thus allowing, for example, for the measurement of the rotational speed of a remote rotating object [5]. The effects of the rotational Doppler shift have also been observed due to the rotational motion of atomic particles [6, 7], in rotational Raman scattering [1] and in nonlinear optics with applications in second harmonic generation [8] and predictions of parametric amplification of light from mechanical rotation [9]. Since the rotational Doppler effect is associated with the angular momentum of the beam [4, 10-12], it will arises with a spin or with orbital angular momentum states. The rotational Doppler effect with orbital angular momentum (OAM) carrying beams has been observed optically [13, 14] and proposed for remote detection of rotation in both terrestrial and astronomical objects [5]. Its effect has been enhanced in specific materials such as ruby where the observed light-dragging effect allows to demonstrate phenomena such as the Mechanical Faraday effect for OAM beams [15] and for structured beams
[16]. These effects were interpreted as the result of slow light propagation in ruby, which in turn has been described as originating from a coherent population oscillation in the medium and a corresponding narrow-band change in the refractive index that can explain the large group refractive indices [17-19]. It is worth noting however, that other models have also been proposed that rely only on the incoherent saturable absorption properties of ruby and lead to similar results without requiring coherent population oscillations within the medium [20]. Both models provide the same intensity dependence for the light dragging effect.

Regardless of the controversy around the details that lead to light-dragging or slow light effects in ruby [20], all proposed models lead to the same conclusion when we consider only the changes in absorption due to a periodic modulation of an intense beam propagating through a saturable-absorbing medium. For example, the incoherent bleaching model [21-23] couples the ground state population with the pump beam intensity evolution and predicts that a weak periodic modulation of the pump beam will modify the saturated absorption coefficient leading to a increasing absorption for increasing modulation frequency. In most experiments, modulation of the pump beam is obtained by direct amplitude modulation of the beam with e.g. an acoustooptic modulator $[17,20]$.

In this work we investigate the effect on an OAM-carrying probe beam of a rotating ruby rod using a Gaussian shaped pump beam. Amplitude modulation of the pump beam is obtained by introducing a probe beam that carries OAM. The probe beam frequency is therefore rotationally Doppler shifted by the ruby rod rotation, thus creating the conditions for absorption modulation as discussed above. Our results therefore show increasing absorption for increasing mechanical rotation speed of the medium, with a rate that increases with increasing OAM of the light beam. This furnishes an additional example of how the rotational Doppler effect combines with light-matter interactions due to external rotation.

An intuitive picture of the rotational equivalent to the Doppler effect is given by considering that the light transmitted in a rotating medium is dragged by that medium [24] giving rise to an angular frequency shift, as happens also to the hands of a clock on a rotating table. In the following we deal with helically-symmetric beams for which the winding number $\ell$ is 


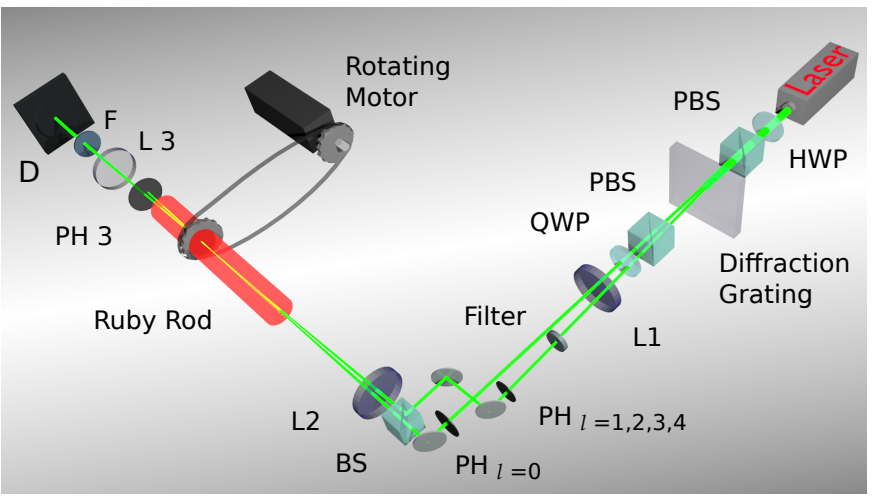

Fig. 1. Schematics of the experimental setup. A CW $532 \mathrm{~nm}$ laser beam passes through a diffraction grating to convert the incident plane wave into transmitted helically phased beams of orbital angular momentum $\ell$ at the $\ell$ order diffracted beam. The $\ell$ state transmitted beam has a diffraction angle of $\Delta \theta=l \cdot \theta$ with $\theta=1^{\circ}$. The two beams are focused down onto the ruby front face and axially transmitted. Then the outcoming probe beam is collected by the detector. The rotation of the ruby rod about its axis is controlled by a brushless rotating motor up to $\pm 60 \mathrm{~Hz}$ by a digital speed control software.

proportional to the OAM. When the beam has both spin and orbital angular momentum, the frequency shift $\Delta \omega$ is given by

$$
\Delta \omega=(\sigma+\ell) \cdot \Omega,
$$

where $\sigma$ and $\ell$ are respectively the spin and the orbital angular momentum of the beam and $\Omega$ is the rotating frequency of the medium [5]. In our experiments we chose to have the pump and the probe with the same circular polarization but different orbital angular momentum, so the total frequency shift between the pump and the probe is

$$
\Delta \omega=\Delta \ell \cdot \Omega,
$$

where $\Delta \ell$ is the difference between the probe and the pump OAM value. By using the beam with OAM $\ell=0$ as an intense pump, we then investigated the effect of a rotating ruby rod on the OAM-carrying probe beam by measuring the transmitted intensity $T$ of the probe as a function of the rotational speed $\Omega$ of the medium. In this case we examine four different values of OAM from $\ell=1$ to $\ell=4$.

The full experimental layout is shown in Fig. 1. A linearly polarised, continuous wave laser with carrier wavelength $(\lambda=$ $532 \mathrm{~nm}$ ) propagates through the half waveplate (HWP) and the polarizing beam splitter (PBS) to control the overall input beam power. Then the light beam propagates through the diffraction grating $D G$ with a fork dislocation centered on the beam axis that converts the Gaussian beam into OAM modes. The diffracted beams pass through a PBS and a quarter waveplate QWP transforms to circular polarisation so as to ensure a rotation-invariant interaction with the birefringent ruby crystal when this rotates. The convex lens $L 1$ of focal length $f=1000 \mathrm{~m}$ focuses the beams and the two pinholes $P H_{\ell=0}$ and $P H_{\ell=1,2,3,4}$ spatially select the pump and the probe beams, respectively. The probe power was set to $70 \mathrm{~mW}$ for all $\ell$ values. Since the OAM probe beams have different sizes for different $\ell$, the pump power was controlled with a variable transmission filter so as to guarantee equal nonlinear interaction for each $\ell$. In order to obtain constant pump intensities in the four OAM states, the pump power was set to
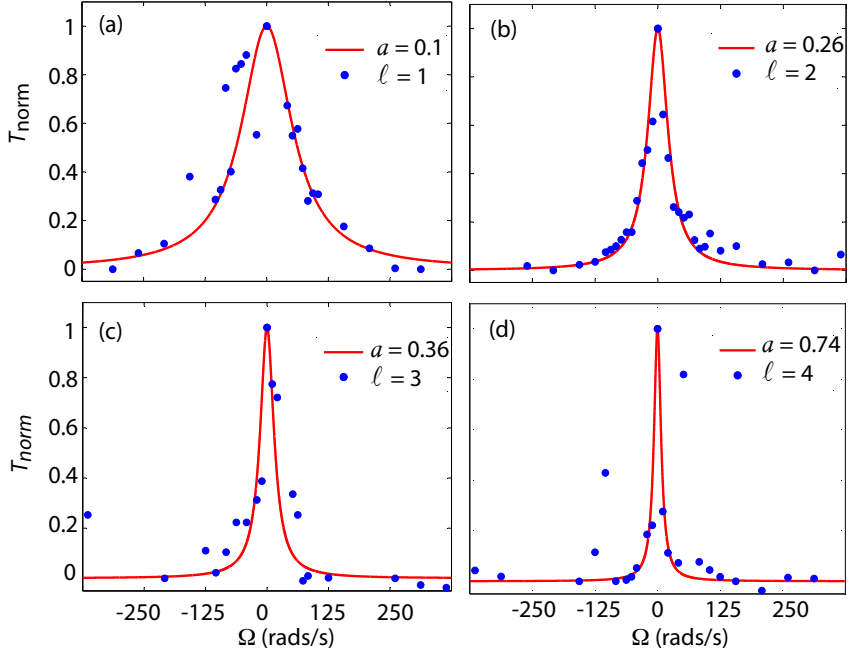

Fig. 2. Normalised relative transmitted intensity $T_{\text {norm }}$ of the probe beam as a function of the rotational speed $\Omega$ of the ruby for four different OAM states: Blue circles - observed transmitted intensity of four different OAM values $\ell=1$ to 4 (a-d), as indicated in the figures. Solid red line - best fits obtained for the Lorentzian phenomenological curve Eq. (5) with value of the parameter $a$ indicated in each graph.

$390 \mathrm{~mW}, 420 \mathrm{~mW}, 561 \mathrm{~mW}$, and $624 \mathrm{~mW}$ for $\ell=1,2,3$ and 4, respectively. The pump and the probe beams are then recombined with a beam splitter $B S$, and are focused down onto the front face of a standard laser ruby crystal (diameter $=10 \mathrm{~mm}$ and length $=90 \mathrm{~mm}$ ) by a $300 \mathrm{~mm}$ focal-length spherical lens $L 2$. The setup is assembled so that the pump and the probe beams are at a small angle yet are spatially overlapped over the whole length of the sample. The brushless rotating motor (RM) allows us to set the speed of the ruby rotation in a range from 0 to 60 $\mathrm{Hz}$ by a digital speed control software and a standard desktop computer. The rotating motor drives the rotation of the ruby by a system of a toothed belt and two gears placed one on the ruby and the other on the motor shaft. The ruby crystal is supported by a high-speed ball bearing system to reduce the friction as it rotates. The pinhole $\mathrm{PH} 3$, blocks the transmitted pump beam at the output and the detector $D$ collects the transmitted probe intensity $I(\Omega)$. The filter $F$ removes any transmitted signal caused by fluorescence in the $600 \mathrm{~nm}$ region.

The experimental results are shown in Fig. 2 where we show the normalised relative transmission, $T_{\text {norm }}$ (i.e. we subtract the minimum transmitted intensity for varying $\Omega$ and then normalise to one) of the probe output intensity for different values of OAM as a function of the rotational speed of the ruby up to $\pm 60 \mathrm{~Hz}$. The rotating ruby medium will rotationally Doppler shift only the probe beam and the sign of the frequency shift depends on the handedness between the OAM state and the rotation direction, we thus investigate both the sidebands $(\Omega>0$ and $\Omega<0)$ by changing the direction of rotation. The results indeed show how the mechanical rotation induces a loss that increases with the magnitude of the mechanical rotation. Moreover, the results show that the absorption rate increases for increasing OAM. As can also be seen in Fig. 2, the transmitted intensity $T_{\text {norm }}$ has an evident Lorentzian-like dependence (red solid lines) on the rotation speed for all the OAM states, a dependence that we qualitatively justify below.

One may intuit the Lorentzian response of our system using 


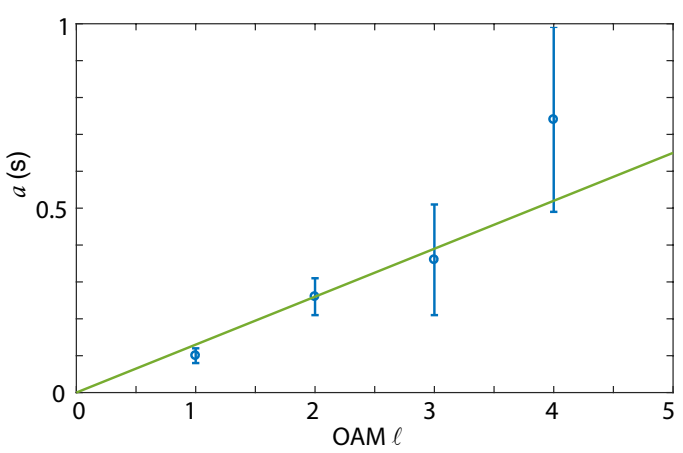

Fig. 3. (Blue squares) Observed values of the decay rate parameter a. According to Eq. (5), the decay rate has a linear behavior in the form $a=$ const $\times \ell$ as a function of the OAM states $\ell$ (Green line). Error bars are indicated with $95 \%$ confidence bounds.

either the coherent-population-oscillations model of Ref. [17] or the incoherent bleaching model of Ref. [20]. In both cases, the small signal gain seen by a weak detuned probe in the presence of a strong pump of intensity $I_{1}$ may be expressed as

$$
\alpha(\Delta \omega)=\frac{\alpha_{0}}{1+I_{0}}-\frac{\alpha_{0} I_{0}}{\left(T_{1} \Delta \omega\right)^{2}+\left(1+I_{0}\right)^{2}},
$$

where $I_{0}=I_{1} / I_{\text {sat }}$ with $I_{\text {sat }}$ the saturation intensity of 1500 $\mathrm{kW} / \mathrm{cm}^{2}$ [25], $\alpha_{0}$ is the unsaturated small-signal gain, and $T_{1}$ the ground state recovery time. Applied to the results in Fig. 2 using a Gaussian pump and probe of OAM $\ell$ we further set $\Delta \omega=\Omega \ell$. Then from Eq. (3) we clearly see that, all other factors being equal, the minimum absorption and peak transmission should occur at zero rotation rate as observed in the experiments. In addition, the second term in Eq. (3) also reveals the Lorentzian dependence on rotation rate that appears in the experiments. However, due to the fact that the beams used in the experiment have transverse intensity profiles, and there is a small angle between the pump and probe beams (meaning that the probe beam is a superposition of OAM states centered on $\ell$ with respect to the pump beam direction [26]), we cannot appeal directly to Eq. (3) to simulate the data. Rather, motivated by Eq. (3) and noting that the second term is small compared to the first for $I_{0} \ll 1$, then the transmitted intensity can be approximated as (neglecting for simplicity in notation the length dependence of $I_{0}(z)$ as this does not change the form of the final result):

$$
\begin{aligned}
I_{T} & =I_{0} e^{-\alpha(\Delta \omega) L} \simeq I_{0} e^{-\frac{\alpha_{0} L}{1+I_{0}}}+\frac{\alpha_{0} L I_{0}^{2}}{\left(T_{1} \Delta \omega\right)^{2}+\left(1+I_{0}\right)^{2}} \\
& \simeq I_{0} e^{-\frac{\alpha_{0} L}{1+I_{0}}}+\frac{\alpha_{0} L I_{0}^{2}}{1+(a \Omega)^{2}},
\end{aligned}
$$

In the measurements, we subtract out the un-modulated contribution $I_{0} e^{-\frac{\alpha_{0} L}{1+I_{0}}}$ and then normalise, thus obtaining the relative normalised transmission, $T_{\text {norm }}$, discussed above and that we approximate with a Lorentzian fit:

$$
T_{\text {norm }}=\frac{1}{1+(a \Omega)^{2}},
$$

where $a=$ const $\times \ell$ depends linearly on the OAM value. This approach is justified by the data as indeed, this is in good agreement with the phenomenological Lorentzian curve. The parameter $a$ quantifies the decay rate for different OAM states: we obtain $a=0.1,0.26,0.36,0.74 \mathrm{~s}$ for $\ell=1,2,3,4$, respectively, demonstrating a differential absorption rate for different OAM probe states induced by the rotation.

Finally, based on Eq. (5) we fit the values of $a$ obtained from the experimental data using a linear function in the form of $a=$ const $\times \ell$. The observed values and the resulting fit is able to fit the data relatively well, as shown in Fig. 3, with const $=0.13 \pm 0.017 \mathrm{~s}$. We also notice an increase in the measurement error for higher $\ell$ that we ascribe to the fact that the higher OAM modes are characterised by a larger ring diameter, thus overlapping with the outer and noisier regions of the Gaussian pump beam.

In summary, we have provided an example of how the rotational Doppler effect can enter into nonlinear light-matter interactions due to external rotation of the medium. This effect leads to a differential absorption rate for different OAM probe states induced by the mechanical rotation of the medium. Considering that the medium is becoming more opaque with the magnitude of the mechanical rotation, this could be used as a method to control the absorption rate of OAM beams in rotating media by the rotational speed and the OAM states and, for example, providing an alternative route to the remote measurement of a spinning object's rotational rate.

\section{REFERENCES}

1. B. A. Garetz, JOSA 71, 609 (1981).

2. M. P. Lavery, S. M. Barnett, F. C. Speirits, and M. J. Padgett, Optica 1, 1 (2014).

3. S. Barreiro, J. Tabosa, H. Failache, and A. Lezama, Phys. Rev. Lett. 97, 113601 (2006).

4. J. Courtial, D. Robertson, K. Dholakia, L. Allen, and M. Padgett, Phys. Rev. Lett. 81, 4828 (1998).

5. M. P. Lavery, F. C. Speirits, S. M. Barnett, and M. J. Padgett, Science. 341, 537 (2013).

6. C. Rosales-Guzmán, N. Hermosa, A. Belmonte, and J. P. Torres, Sci. Rep. 3, 2815 (2013).

7. L. Allen, M. Babiker, and W. Power, Opt. Commun. 112, 141 (1994).

8. G. Li, T. Zentgraf, and S. Zhang, Nat. Phys. 12, 736 (2016).

9. D. Faccio and E. Wright, Phys. Rev. Lett. 118, 093901 (2017).

10. M. Padgett, G. Whyte, J. Girkin, A. Wright, L. Allen, P. Öhberg, and S. M. Barnett, Opti. Lett. 31, 2205 (2006).

11. L. Allen and M. Padgett, J. Mod. Opt. 54, 487 (2007).

12. J. B. Götte, S. M. Barnett, and M. Padgett, "On the dragging of light by a rotating medium," in "Proc. Roy. Soc. Lon. A," , vol. 463 (The Royal Society, 2007), vol. 463, pp. 2185-2194.

13. J. Courtial, K. Dholakia, D. Robertson, L. Allen, and M. Padgett, Phys. Rev. Lett. 80, 3217 (1998).

14. F. C. Speirits, M. P. Lavery, M. J. Padgett, and S. M. Barnett, Opt. Lett. 39, 2944 (2014).

15. E. Wisniewski-Barker, G. M. Gibson, S. Franke-Arnold, R. W. Boyd, and M. J. Padgett, Opt. Exp. 22, 11690 (2014).

16. E. Wisniewski-Barker, G. Gibson, S. Franke-Arnold, Z. Shi, R. W. Boyd, and M. J. Padgett, New J. Phys. 15, 083020 (2013).

17. M. S. Bigelow, N. N. Lepeshkin, and R. W. Boyd, Phys. Rev. Lett. 90, 113903 (2003).

18. R. W. Boyd and D. J. Gauthier, Science. 326, 1074 (2009).

19. M. S. Bigelow, N. N. Lepeshkin, and R. W. Boyd, Science. 301, 200 (2003).

20. B. Macke, I. Razodobreev, and B. Ségard, Phys. Rev. A 95, 063830 (2017).

21. F. Gires and F. Combaud, J. Phys. (Paris) 26, 325 (1965).

22. A. Selden, Br. J. Appl. Phys. 18, 743 (1967).

23. B. Macke and B. Ségard, Phys. Rev. A 78, 013817 (2008).

24. R. V. Jones, Proc. Roy. Soc. Lond. 349, 423 (1976).

25. P. Liao and D. M. Bloom, Opt. letters 3, 4 (1978). 
26. T. Roger, J. J. Heitz, E. M. Wright, and D. Faccio, Sci. reports 3, 3491 (2013).

\section{REFERENCES}

1. B. A. Garetz, "Angular doppler effect", JOSA 71, 609-611 (1981).

2. M. P. Lavery, S. M. Barnett, F. C. Speirits, and M. J. Padgett, "Observation of the rotational Doppler shift of a white-light, orbital-angularmomentum-carrying beam backscattered from a rotating body", Optica 1, 1 (2014).

3. S. Barreiro, J. Tabosa, H. Failache, and A. Lezama, "Spectroscopic observation of the rotational Doppler effect", Phys. Rev. Lett. 97, 113601 (2006).

4. J. Courtial, D. Robertson, K. Dholakia, L. Allen, and M. Padgett, "Rotational frequency shift of a light beam", Phys. Rev. Lett. 81, 4828 (1998).

5. M. P. Lavery, F. C. Speirits, S. M. Barnett, and M. J. Padgett, "Detection of a spinning object using light's orbital angular momentum", Science. 341, 537 (2013).

6. C. Rosales-Guzmán, N. Hermosa, A. Belmonte, and J. P. Torres, "Experimental detection of transverse particle movement with structured light", Sci. Rep. 3, 2815 (2013).

7. L. Allen, M. Babiker, and W. Power, "Azimuthal Doppler shift in light beams with orbital angular momentum", Opt. Commun. 112, 141 (1994).

8. G. Li, T. Zentgraf, and S. Zhang, "Rotational Doppler effect in nonlinear optics", Nat. Phys. 12, 736 (2016).

9. D. Faccio and E. Wright, "Nonlinear Zel'dovich effect: parametric amplification from medium rotation", Phys. Rev. Lett. 118, 093901 (2017).

10. M. Padgett, G. Whyte, J. Girkin, A. Wright, L. Allen, P. Öhberg, and S. M. Barnett, "Polarization and image rotation induced by a rotating dielectric rod: an optical angular momentum interpretation", Opti. Lett. 31, 2205 (2006).

11. L. Allen and M. Padgett, "Equivalent geometric transformations for spin and orbital angular momentum of light", J. Mod. Opt. 54, 487 (2007).

12. J. B. Götte, S. M. Barnett, and M. Padgett, "On the dragging of light by a rotating medium", in "Proc. Roy. Soc. Lon. A," J. Mod. Opt. 463, 2185-2194 (The Royal Society, 2007).

13. J. Courtial, K. Dholakia, D. Robertson, L. Allen, and M. Padgett, "Measurement of the rotational frequency shift imparted to a rotating light beam possessing orbital angular momentum", Phys. Rev. Lett. 80, 3217 (1998).

14. F. C. Speirits, M. P. Lavery, M. J. Padgett, and S. M. Barnett, "Optical angular momentum in a rotating frame", Opt. Lett. 39, 3944 (2014).

15. E. Wisniewski-Barker, G. M. Gibson, S. Franke-Arnold, R. W. Boyd, and M. J. Padgett, "Mechanical Faraday effect for orbital angular momentum-carrying beams", Opt. Exp. 22, 11690 (2014).

16. E. Wisniewski-Barker, G. Gibson, S. Franke-Arnold, Z. Shi, R. W. Boyd, and M. J. Padgett, "Evidence of slow-light effects from rotary drag of structured beams", New J. Phys. 15, 083020 (2013).

17. M. S. Bigelow, N. N. Lepeshkin, and R. W. Boyd, "Observation of ultraslow light propagation in a ruby crystal at room temperature", Phys. Rev. Lett. 90, 113903 (2003).

18. R. W. Boyd and D. J. Gauthier, "Controlling the velocity of light pulses", Science 326, 1074 (2009).

19. M. S. Bigelow, N. N. Lepeshkin, and R. W. Boyd, "Superluminal and slow light propagation in a room-temperature solid", Science 301, 200 (2003).

20. B. Macke, I. Razodobreev, and B. Ségard, "Slow light in saturable absorbers: progress in the resolution of s controversy", Phys. Rev. A 95, 063830 (2017).

21. F. Gires and F. Combaud, "Saturation de l'absorption optique de certains solutions de phtalocyanines", J. Phys. (Paris) 26, 325 (1965).

22. A. Selden, "Pulse transmission through a saturable absorber", Br. J. Appl. Phys. 18, 743 (1967).

23. B. Macke and B. Ségard, "Slow light in saturabale absorbers", Phys. Rev. A 78, 013817 (2008).

24. R. V. Jones, "Rotary aether drag", Proc. Roy. Soc. Lond. 349, 423 (1976).

25. P. Liao and D. M. Bloom, "Continuous-wave backward-wave generation by degenerate four-wave mixing in ruby", Opt. Lett. 3, 4 (1978).

26. T. Roger, J. J. Heitz, E. M. Wright, and D. Faccio, "Non-collinear inter- 
action of photons with orbital angular momentum", Sci. Rep. 3, 3491

(2013). 